\title{
EDITORIAL
}

\section{High risk for schizophrenia: genetic considerations ${ }^{1}$}

Being the identical twin of a schizophrenic, as Meehl (1962) pointed out, is the best single predictor of schizophrenia, but such twins are hardly appropriate subjects for prospective high-risk studies. Some monozygotic co-twins of schizophrenics will already be affected when the case is discovered. Unless ascertained soon after the onset of the first twin's illness, the discordant partners are likely to be no longer at high risk, since most pairs that become concordant do so within a few years. They would not be of much help in detecting early signs of disorder or making predictions about their response to environmental changes. Furthermore, some pairs may be discordant because the illness in the proband was a symptomatic schizophrenia. Studied retrospectively, however, they remain of value in the search for stable constitutional traits predisposing to schizophrenia, as exemplified in the approach of Wyatt et al. (1973). Such a trait should in general distinguish schizophrenics from non-schizophrenics but should occur in both affected and unaffected members of discordant $\mathrm{MZ}$ pairs.

The offspring of two schizophrenic parents also incur a risk of developing schizophrenia - usually taken to be around $40 \%$ - which places them, like MZ co-twins, within the high risk range of $25 \%$ or over, using the term in the sense in which it is generally employed by geneticists when speaking of recurrence risks in genetic disease. In their risk study Erlenmeyer-Kimling (1975) and her colleagues have been able to include a group of 13 such children, aged 7-12 at first contact, together with 9 of their siblings.

The great majority of the high-risk studies which have burgeoned recently (Garmezy, 1974) examine children with one schizophrenic parent, since they are more accessible. On average they have only a moderately high risk of around $10 \%$ of becoming affected if they live long enough. Up till recently, the range varied from $7 \%$ to $17 \%$ in different studies, and the risk depends to some extent on the type of schizophrenia in the proband and the characteristics of the spouse. Though schizophrenia cannot be called a genetic disease in the same sense as Huntington's chorea or cystic fibrosis, it has been argued from the results in family, twin and adoption studies that genetic factors are the most uniformly potent cause (Gottesman \& Shields, 1972). No environmental indicator predicts a raised risk of schizophrenia in small or moderate-sized samples of persons not already known to be genetically related to a schizophrenic. What, then, can we hope to learn from prospective highrisk work about the developmental genetic aspects of schizophrenia and about gene-environment interaction that cannot be discovered by other, simpler methods?

The children of schizophrenics are not uniformly at risk. Whichever model of inheritance is preferred, they will be genetically heterogeneous, with some of them at much higher risk for schizophrenia than others. On a simple monogenic theory half of them should be at no increased risk at all. Prospective studies might offer a better chance of recognizing different phenotypic expressions of various genotypes than retrospective studies where it is difficult or impossible to assess reliably the premorbid condition of an active or remitted schizophrenic. This is most obviously so as regards developmental characteristics and psychophysiological, attentional and other experimental psychological measures. A combination of suspect characteristics in the same person would be suggestive. Hanson et al. (1976) made use of longitudinal data collected in a study of child development. They found that the 30 children of schizophrenic parents from Minneapolis were remarkably normal on a host of perinatal, neurological and psychometric variables. However, of the 116 experimental and control children all 5 who had a combination of poor motor skills, large intra-individual variance on

1 Address for correspondence: Dr James Shields, Institute of Psychiatry, De Crespigny Park, Denmark Hill, London SE5 8AF. 
intelligence tests, and ratings of 'schizoid' behaviour at the ages of 4 and 7 were the children of schizophrenic parents. It was predicted that these 5 are especially vulnerable to future schizophrenia.

Besides any information which might accrue concerning early or modifiable signs of the developing illness, there is the possibility of leads to fundamental disturbances of psychological functioning that might be thought to be closer to what was inherited in schizophrenia than the psychosis itself. By looking at characteristics which resemble those of schizophrenics and studying them in the relatives of schizophrenics and in control groups, some of the risk studies should be maximizing their chances of finding any such behavioural characteristics of schizoidia - if they exist (Shields et al. 1975).

To find out whether any such characteristics are more clearly inherited than schizophrenia, one would need to carry out genetic studies of them in schizophrenics' families and in the general population. The incidence of schizophrenia in the families of persons from the general population found to be deviant in such characteristics would also be needed in order to see whether they have any general bearing on the predisposition to schizophrenia. For example, if it were discovered that fidgetiness in childhood was a good predictor or early manifestation of Huntington's chorea in Huntington families, this would not imply that fidgety children in other families were at risk for this rare disease. There is no special reason to suppose that a style of thinking, a perceptual anomaly or a physiological measure will be any more simply inherited or less heterogeneous in its aetiology than schizophrenia itself. One might, however, be fortunate enough to identify a normal behavioural variable which at one of its extremes contributed to some extent to the predisposition to schizophrenia along with other perhaps more specific factors (Smith \& Mendell, 1974). Shorter-term longitudinal studies and cross-sectional studies at different ages should shed light on which characteristics are age-dependent and which remain stable indicators of high risk. Stable traits are clearly of greater value for genetic investigations than state-dependent ones, and prospective studies may help identify them.

On the environmental side the hope is that a variable of one kind or another may be identified to which those genetically at risk for schizophrenia are particularly sensitive and which offers scope for preventive intervention. Perinatal factors and the nature of the final precipitating events are as easily investigated retrospectively as prospectively, but it would be interesting to have premorbidly made assessments of the quality of family life and social adjustment.

Follow-up information is the ultimate test of any claim to have identified individuals at highest risk genetically, or a genetic trait in the population which is correlated with the predisposition to schizophrenia, or an interacting environmental factor which offers scope for prevention. One of the studies described by Garmezy (1974), that of McNeil \& Kaij, statts as early as the eighth month of pregnancy with recordings of the foetal heart beat for orienting response and habituation. Obviously it will not be feasible to follow such subjects through to the final life event before the onset in those who become schizophrenic, and then on to an age where the healthy offspring are all too old to be any longer at risk.

An inevitable drawback from the genetic point of view of studying the children of schizophrenics prospectively is that only a minority of schizophrenics have schizophrenic parents, in particular parents who were already schizophrenic when the children were born. The earlier the age at which the high risk children are ascertained, the greater the proportion exposed from an early age to environmental circumstances not experienced by most schizophrenics. Rearing by psychotic and other sick parents (Rutter, 1966) can lead to behaviour disturbances in children for environmental reasons. ${ }^{2}$ Childhood differences found between high-risk and low-risk groups may therefore not reflect an increased schizophrenia risk but relate to the quality or lack of family life which is hard to match experimentally. Furthermore, not all schizophrenics have marked childhood or adolescent problems. Many of the experimental variables studied which are thought to indicate increased schizophrenia risk, such as unstable psychophysiology or disordered attention, may be non-specific for schizo-

\footnotetext{
2 Younger sibs of schizophrenics do not suffer this particular disadvantage but have other limitations as subjects for prospective studies. They are more often used as controls than as persons with a moderately high risk themselves.
} 
phrenia and in addition present the all too common problem of unreliable measurement. It is therefore particularly desirable to discover which offspring have schizophrenic outcomes. On investigation at successive ages one might expect the composition of the sick group among high-risk offspring to change, some subjects with behaviour disorders dropping out or showing themselves to be nonschizophrenic, and others, including definite schizophrenics, being added.

The current enthusiasm for high-risk studies is due more than anything to the imaginative and productive work of Mednick and Schulsinger in Copenhagen. Thanks to the facilities for and attitudes towards genetic and epidemiological research in the Scandinavian countries, these workers would appear to be the first who might come near to approaching these seemingly unrealistic followup requirements. This is not the place to describe their projects in detail. Many of their earlier papers are republished in Mednick et al. (1974) and are succinctly described by Schulsinger \& Jacobsen (1975). Currently their hypothesis is that potential schizophrenics have an autonomic nervous system that responds too quickly and too much to 'unkind environments'; an abnormally fast electrodermal recovery rate, which 'may be an important part of the genetic pattern transmitted from parent to child'; and a particular sensitivity to certain types of perinatal stress. Their psychophysiological findings have apparently not been confirmed by van Dyke et al. (1974) on the adopted-away children of schizophrenics or by Erlenmeyer-Kimling (1975) even when both parents were schizophrenic. This may be partly due to considerations such as age, diagnosis and other characteristics of the sample or to testing procedures. From their later work (Mirdal et al. 1974) and the careful work of McNeil \& Kaij $(1973,1974)$ in Sweden, the association with perinatal factors, if it exists, may be much more slender than was originally thought. Their extensive data have only been partly analysed; and many of their conclusions - and further studies - have been largely based on the results of the first 20 sick children in their high-risk group identified in 1967. Their prospective study of 3-year-old Mauritian children with abnormal psychophysiology, some attending special nursery schools and others not (Schulsinger et al. 1975), should be interesting for what it can tell us about the stability of psychophysiology and its relation to behaviour problems and environment, but it would be premature to suppose that it has much specific bearing on the prevention of schizophrenia.

Nevertheless, the Mednick-Schulsinger team (Hanne Schulsinger, 1976) have followed up and, in 1972, succeeded in interviewing and testing a high proportion of their 207 high-risk and 104 low-risk subjects originally studied in 1962. At follow-up they were aged between 20 and 30 (mean approaching 24). The investigators themselves admit to 'a low threshold for diagnostic labelling', since they 'did not want to exclude even minimal psychopathological manifestations' in either group. However, in the high-risk group the number of definite schizophrenics identified blindly by procedures which included the PSE (10 according to CATEGO, 15 according to a consensus diagnosis) was not unexpectedly high; and there was only one schizophrenic among the low-risk controls.

It is therefore with great interest that one waits the detailed report of the extensive social, psychological and psychophysiological information collected in 1962 and 1972, to see how it relates to clinical state at an age when the group has survived nearly half the risk period for schizophrenia. But in view of the inherent difficulties and the rather slender relation found in earlier longitudinal studies (e.g. Hagnell, 1966) between previous personality assessments and subsequent breakdown, it may be unwise to set expectations very high. Schizophrenia is likely to be too diverse in aetiology for the recognition of a premorbid schizophrenic personality or a single biological characteristic that could be equated with the genotype. Yet one hopes the efforts of the Mednick and Schulsinger and other high risk studies will at least succeed in identifying and relating to one another some of the multiple causes in the development of many schizophrenias. 


\section{REFERENCES}

Erlenmeyer-Kimling, L. (1975). A prospective study of children at risk for schizophrenia: methodological considerations and some preliminary findings. In Life History Research in Psychopathology, vol. 4 (ed. R. D. Wirt, G. Winokur \& M. Roff). University of Minnesota Press: Minneapolis.

Garmezy, N. (1974). Children at risk: The search for the antecedents of schizophrenia. Schizophrenia Bulletin 8, $14-90 ; 9,55-125$.

Gottesman, I. I. \& Shields, J. (1972). Schizophrenia and Genetics: a Twin Study Vantage Point. Academic Press: New York.

Hagnell, O. (1966). A Prospective Study of the Incidence of Mental Disorder. Scandinavian University Books: Stockholm.

Hanson, D. R., Gottesman, I. I. \& Heston, L. L. (1976). Some possible childhood indicators of adult schizophrenia inferred from children of schizophrenics. British Journal of Psychiatry 129, 142-154.

McNeil, T. F. \& Kaij, L. (1973). Obstetric complications and physical size of offspring of schizophrenic, schizophreniclike, and control mothers. British Journal of Psychiatry 123, 341-348.

McNeil, T. F. \& Kaij, L. (1974). Reproduction among female mental patients: obstetric complications and physical size of offspring. Acta Psychiatrica Scandinavica 50, 3-15.

Mednick, S. A., Schulsinger, F., Higgins, J. \& Bell, B. (eds.) (1974). Genetics, Environment and Psychopathology. NorthHolland: Amsterdam.

Meehl, P. E. (1962). Schizotaxia, schizotypy, schizophrenia. American Psychologisı 17, 827-838.
Mirdal, G. K. M., Mednick, S. A., Schulsinger, F. \& Fuchs, F. (1974). Perinatal complications in children of schizophrenic mothers. Acta Psychiatrica Scandinavica 50, 553-568.

Rutter, M. (1966). Children of Sick Parents: an Environmental and Psychiatric Study. Maudsley Monograph no. 16. Oxford University Press: London.

Schulsinger, F. \& Jacobsen. B. (1975). The heredity-environment issue in psychiatry. Perspectives from research at the Psykologisk Institut, Department of Psychiatry, Kommunehospitalet. Acta Psychiatrica Scandinavica Supplement 261, pp. 44-58.

Schulsinger, F., Mednick, S. A., Venables, P. H., Raman, A. C. \& Bell, B. (1975). Early detection and prevention of mental illness: the Mauritius project. Neuropsychobiology 1, 166-179.

Schulsinger, H. (1976). A ten-year follow-up of children of schizophrenic mothers: clinical assessment. Acta Psychiatrica Scandinavica 53, 371-386.

Shields, J., Heston, L. L. \& Gottesman, I. I. (1975). Schizophrenia and the schizoid: the problem for genetic analysis. In Genetic Research in Psychiatry (ed. R. R. Fieve, D. Rosenthal \& H. Brill). Johns Hopkins University Press: Baltimore and London.

Smith, C. \& Mendell, N. R. (1974). Recurrence risks from family history and metric traits. Annals of Human Generics, London 37, 275-286.

van Dyke, J. L., Rosenthal, D. \& Rasmussen, P. V. (1974). Electrodermal functioning in adopted-away offspring of schizophrenics. Journal of Psychiatric Research 10, 199215.

Wyatt, R. J., Murphy, D. L., Belmaker, R., Cohen, S., Donnelly, C. H. \& Pollin, W. (1973). Reduced monoamine oxidase activity in platelets: a possible genetic marker for vulnerability to schizophrenia. Science 179, 916-918. 